\title{
ON THE EXISTENCE OF SOLUTIONS OF THE HELIUM WAVE EQUATION
}

\author{
BY \\ TOSIO KATO
}

Introduction. The helium wave equation has been studied by several authors $\left({ }^{1}\right)$ with a view to obtaining the exact solution for the ground state, or at least to establishing its existence. The subject is of importance as a matter of principle, since it implies the decision whether the present formulation of nonrelativistic many-body problems is correct $\left({ }^{2}\right)$. Also, the validity of the variational method $\left({ }^{3}\right)$ depends upon it, for this method would be meaningless if there existed no stationary state at all.

Unfortunately the attempts of these authors have not been successful; their method of series expansion proved to be powerless to control manyparticle problems.

In the present paper, the writer wishes to settle this problem by showing rigorously that the wave equation for the two-electron problem, in particular the helium wave equation, has a very large number-even infinity if the nucleus is assumed to be infinitely heavy-of solutions corresponding to stationary states of the system. In particular the existence of the ground state solution is established.

Our method is different from those adopted by the authors cited above. We shall not attempt, for the present, to find explicit expressions for the solutions and shall resort to a method based on the abstract theory of operators in Hilbert space. The essential part of our theory may be regarded as already completed in the previous paper $\left({ }^{4}\right)$, where it was shown that the Hamiltonian operator of every atom, molecule, or ion is essentially self$\operatorname{adjoint}\left({ }^{5}\right)$. This means that the closed Hamiltonian $\tilde{H}$ of such a system, which is uniquely determined by the given Hamiltonian as a formal differential operator, is self-adjoint in the strict sense, that is, that it has a complete set (discrete or continuous) of eigenfunctions. Further, it was shown that eigen-

Received by the editors October 15, 1948.

(1) J. H. Bartlett, J. J. Gibbons, and C. G. Dunn, Physical Review vol. 47 (1935) p. 679; T. H. Gronwall, Physical Review vol. 51 (1937) p. 655; J. H. Bartlett, Physical Review vol. 51 (1937) p. 661 ; A. S. Coolidge and H. M. James, Physical Review vol. 51 (1937) p. 855; E. C. Kemble, The fundamental principles of quantum mechanics, International Series in Physics, New York and London, 1937, pp. 208-212.

(2) J. H. Bartlett, J. J. Gibbons, and C. G. Dunn, footnote 1.

(3) E. A. Hylleraas, Zeitschrift für Physik vol. 54 (1929) p. 347; vol. 65 (1930) p. 209.

(4) T. Kato, Fundamental properties of Hamiltonian operators of Schrödinger type, Trans. Amer. Math. Soc. vol. 70 (1951) pp. 195-211. This paper will be referred to as I.

(5) $\mathrm{I}$, in particular, introduction. 
functions $\left(^{6}\right)$ of $\tilde{H}$ are, if they exist, sufficiently differentiable (even analytic) and are solutions of the wave equation considered as a differential equation, except at singular points of the potential [I, §8].

The only one thing left to be proved in the present paper is, therefore, the existence of these eigenfunctions, or what comes to the same thing, the existence of eigenvalues of $\widetilde{H}\left({ }^{7}\right)$. This part of our theory alone is dependent on the number of particles, and is easily carried out in the case of the two-electron system by making use of known results concerning the spectrum of hydrogen-like atoms. The proof given below is rather in the nature of rough estimation of eigenvalues, and has nothing direct to do with the complicated differential equation. The result would be much improved by more detailed calculation. It will be noted here that, although there might be formally nothing new in this part of our theory $\left({ }^{8}\right)$, it assures the existence of solutions of the wave equation only when we know the result of the previous paper $\left({ }^{9}\right)$.

Clearly our method does not inform us of the behavior of the wave functions at singular points of the potential. Once their existence is established, however, we are not without means to study their behavior at such points. For instance, we can show that they are still bounded at those singular points. But we shall not enter into these details in this paper.

It seems that our method should be preferred to the classical one such as series expansion, for the existing analytical tools are too weak to permit us a frontal attack in that sense even in the simplest case of many-particle problems. If we pass to problems of three or more electrons, the classical method is entirely out of question, whereas our method might be expected to be successful if we carry out more detailed calculations than those given in this paper, which are indeed quite rough ones.

1. Principle of the method. According to the result obtained in the previous paper $\left({ }^{10}\right)$, the closed Hamiltonian $\tilde{H}$ of every atom is a self-adjoint operator( $\left.{ }^{11}\right)$ in the Hilbert space $\mathfrak{S}$ consisting of all quadratically integrable functions defined in the configuration space. It means that $\widetilde{H}$ can be "diagonalized" as $\left({ }^{12}\right)$

$$
\tilde{H}=\int_{-\infty}^{+\infty} \lambda d E(\lambda) .
$$

(6) In the following we mean by an eigenvalue a discrete one, and similarly for an eigenfunction, if the contrary is not positively stated.

(7) Cf. footnote 11 of I.

(8) In fact, it can be regarded as the zero-approximation in the variational method, see $\$ 5$.

$\left({ }^{9}\right)$ I, Introduction, $\$ 8$.

(10) I, $\$ 6$ and introduction.

(11) M. H. Stone, Linear transformations in Hilbert space, Amer. Math. Soc. Colloquium Publications, vol. 15, New York, 1932, p. 50.

(12) Footnote 11, chap. V, J. v. Neumann, Mathematische Grundlagen der Quantenmechanik, Berlin, Springer, 1932, p. 61. 
This is a convenient expression comprising the continuous as well as the discrete spectrum. $E(\lambda)$ is called a resolution of the identity and is a family of projection operators with the following properties $\left({ }^{12}\right)$

1. $E(\lambda) E(\mu)=E(\operatorname{Min}(\lambda, \mu))$,

2. $E(\lambda+\epsilon) \rightarrow E(\lambda)(\epsilon \rightarrow 0, \epsilon>0)$,

3. $E(\lambda) \rightarrow 0(\lambda \rightarrow-\infty), E(\lambda) \rightarrow 1(\lambda \rightarrow+\infty)$.

As yet we do not know whether $\widetilde{H}$ really possesses those eigenvalues which the experimentation has disclosed. But if we can show that

$$
N \leqq \operatorname{dim} E(\mu)<+\infty\left({ }^{13}\right)
$$

for some positive integer $N$ and a real number $\mu, E(\lambda)$ can change only discontinuously in the interval $-\infty<\lambda \leqq \mu$ and there are at most a finite number of eigenvalues $\left({ }^{14}\right)$ and no continuous spectrum, while there are at least $N$ eigenvalues in that interval $\left({ }^{15}\right)$.

This is the principle of our method. We shall show that in the case of the two-electron problem, we can find $N$ and $\mu$ satisfying (1) and such that $N$ can be made very large by suitable choice of $\mu$, even as large as we like if the nucleus is assumed to be infinitely heavy.

For this purpose, we make use of the following general lemmas.

LEMMA 1. Let $H$ be any self-adjoint operator with the domain $\mathcal{D}_{H}$ and let $E(\lambda)$ be the corresponding resolution of the identity. If there is an $N$-dimensional linear manifold $\mathfrak{M}_{N} \subseteq \mathcal{D}_{H}$ such that $(H f, f) \leqq \mu(f, f)$ for every $f \in \mathfrak{M}_{N}$, then $\operatorname{dim} E(\mu) \geqq N$.

Proof. If $\operatorname{dim} E(\mu)<N$ contrary to the assertion, there would be an $f \in \mathfrak{M}_{N}(f \neq 0)$ which is orthogonal to all elements of the range of $E(\mu)$, that is, $E(\mu) f=0$. It follows from the spectral formula that $(H f, f)>\mu(f, f)$, contrary to the hypothesis.

LEMMA 2. Let $H$ and $H^{\prime}$ be two self-adjoint operators with the domains $\mathcal{D}_{H}$ and $\mathcal{D}_{H^{\prime}}$, and let $E(\lambda)$ and $E^{\prime}(\lambda)$ be the corresponding resolutions of the identity. If $\mathcal{D}_{H} \subseteq \mathcal{D}_{H^{\prime}}$ and $(H f, f) \geqq\left(H^{\prime} f, f\right)$ for every $f \in \mathcal{D}_{H}$, then $\operatorname{dim} E(\lambda)$ $\leqq \operatorname{dim} E^{\prime}(\lambda)$ for every $\lambda$.

Proof. Let $\mathfrak{M}_{N}$ be the range of $E(\lambda)-E\left(\lambda_{1}\right)\left(\lambda>\lambda_{1}\right)$. Then $\mathfrak{M}_{N} \subseteq \mathcal{D}_{H}$, and it follows from the spectral formula that $(H f, f) \leqq \lambda(f, f)$ for every $f \in \mathfrak{M}_{N}$. Hence we have by hypothesis $\left(H^{\prime} f, f\right) \leqq \lambda(f, f)$ for every $f \in \mathfrak{M}_{N}$. Application of Lemma 1 to the operator $H^{\prime}$ yields $\operatorname{dim} E^{\prime}(\lambda) \geqq N=\operatorname{dim}\left(E(\lambda)-E\left(\lambda_{1}\right)\right)$. Making $\lambda_{1} \rightarrow-\infty$, we obtain $\operatorname{dim} E^{\prime}(\lambda) \geqq \operatorname{dim} E(\lambda)$.

2. First estimation. As we have shown $[I, \S 6]$ the closed Hamiltonian $\tilde{H}$ of the two-electron problem is given by

( $\left.{ }^{13}\right) \operatorname{dim} E(\mu)$ is the dimension number of the range of $E(\mu)$.

(14) A degenerate eigenvalue is always counted repeatedly.

(15) $\operatorname{dim} E(\mu)<+\infty$ is essential, for otherwise there might be no eigenvalue at all, the spectrum being entirely continuous. 


$$
\widetilde{H}=p_{1}^{2}+p_{2}^{2}+2 \alpha p_{1} \cdot p_{2}-\frac{2}{r_{1}}-\frac{2}{r_{2}}+\frac{2}{Z r_{12}} .
$$

Here we used a system of units slightly different from usual atomic units $\left({ }^{16}\right)$, $Z$ is the atomic number, and $\alpha$ is given by

$$
\alpha=m /(M+m), \quad 0<\alpha<1,
$$

where $m$ and $M$ are the electronic and the nuclear mass respectively. (2) can also be written in the form

$$
\widetilde{H}=(1-\alpha)\left(p_{1}^{2}+p_{2}^{2}\right)+\alpha\left(p_{1}+p_{2}\right)^{2}-\frac{2}{r_{1}}-\frac{2}{r_{2}}+\frac{2}{Z r_{12}} .
$$

The domain $\mathcal{D}_{0}$ of $\tilde{H}$ was shown to be the set of all functions $f\left(\boldsymbol{r}_{1}, \boldsymbol{r}_{2}\right)$ whose representations $F\left(\boldsymbol{p}_{1}, \boldsymbol{p}_{2}\right)$ in momentum space satisfy the conditions [I, equation (11)]

$$
\int\left|F\left(p_{1}, p_{2}\right)\right|^{2} d p_{1} d p_{2}<\infty, \quad \int p_{i}^{4}\left|F\left(p_{1}, p_{2}\right)\right|^{2} d p_{1} d p_{2}<\infty \quad(i=1,2) .
$$

These are precisely the conditions expressing that the kinetic energy operator $T_{0}=p_{1}^{2}+p_{2}^{2}+2 \alpha p_{1} \cdot p_{2}$ can be applied to $f[\mathrm{I}, \S 2]$. It was further shown that the potential energy operator is defined for all these functions [I, §5] and that the domain $\mathscr{D}_{0}$ of the total Hamiltonian $\widetilde{H}$ is identical with that of $T_{0}$ and independent of the potential $[I, \S \S 6,9]$. In particular $\mathscr{D}_{0}$ is independent of $\alpha$ and $Z$.

First we compare $\widetilde{H}$ with the operator

$$
H^{\prime}=(1-\alpha)\left(p_{1}^{2}+p_{2}^{2}\right)-\frac{2}{r_{1}}-\frac{2}{r_{2}} .
$$

According to what we have just stated, $H^{\prime}$ is also self-adjoint with the same domain $\mathcal{D}_{0}$. Since by (3)

$$
\tilde{H}=H^{\prime}+\alpha\left(p_{1}+p_{2}\right)^{2}+\frac{2}{Z r_{12}},
$$

it follows that $(\tilde{H} f, f) \geqq\left(H^{\prime} f, f\right)$ for every $f \in \mathcal{D}_{0}$. It follows from Lemma 2 that

$$
\operatorname{dim} E(\lambda) \leqq \operatorname{dim} E^{\prime}(\lambda) \quad(-\infty<\lambda<+\infty)\left({ }^{17}\right),
$$

where $E^{\prime}(\lambda)$ is the resolution of the identity ${ }^{(2)}$ belonging to $H^{\prime}$.

Now the nature of the spectrum of $H^{\prime}$ is evident, for $H^{\prime}$ is the Hamiltonian of a system composed of two independent hydrogen-like atoms. Since

${ }^{(16)}$ See, for example, Kemble, footnote 1, p. 420.

(17) It is essential here that the domains of $\widetilde{H}$ and $H^{\prime}$ are the same. 
the eigenvalues of the operator $p^{2}-2 \beta r^{-1}$ for a hydrogen-like atom are given by $-\beta^{2} n^{-2}$ with multiplicities $n^{2}(n=1,2, \cdots)\left({ }^{18}\right)$, it follows that the lowest eigenvalues of $H^{\prime}$ are given by

$$
-\frac{1}{1-\alpha}\left(1+\frac{1}{n^{2}}\right) \quad(n=1,2, \cdots)
$$

with multiplicites $2 n^{2}$ for $n \geqq 2$ and 1 for $n=1$, and that the spectrum of $H^{\prime}$ contains no other points in the interval $\lambda<-(1-\alpha)^{-1}$. In other words we have $\operatorname{dim} E^{\prime}(\lambda)<\infty$ and hence by (4)

$$
\operatorname{dim} E(\lambda)<\infty \quad \text { if } \lambda<-(1-\alpha)^{-1} .
$$

3. Second estimation. We rewrite (2) in the following form:

$$
\begin{aligned}
\widetilde{H} & =H_{1}+H_{2}+W, \\
H_{1} & =p_{1}^{2}-2 r_{1}^{-1}, \\
H_{2} & =p_{2}^{2}-2\left(1-Z^{-1}\right) r_{2}^{-1}, \\
W & =2 \alpha p_{1} \cdot p_{2}+2 Z^{-1}\left(r_{12}^{-1}-r_{2}^{-1}\right) .
\end{aligned}
$$

If we regard $H_{1}$ as an operator of the one-electron problem, it has eigenvalues $-n^{-2}(n=1,2, \cdots)\left({ }^{18}\right)$. The normalized wave function for the ground state is $\left({ }^{19}\right) \phi\left(\boldsymbol{r}_{1}\right)=\pi^{-1 / 2} e^{-r_{1}}$. Similarly $H_{2}$ has eigenvalues

$$
-\left(1-Z^{-1}\right)^{2} n^{-2} \quad(n=1,2, \cdots)
$$

and corresponding eigenfunctions

$$
\psi_{n l m}\left(\boldsymbol{r}_{2}\right) \quad(n=1,2, \cdots ; l=0,1, \cdots, n-1 ; m=0, \pm 1, \cdots, \pm l),
$$

where we denote as usual by $n, l, m$ the total, azimuthal, and magnetic quantum number respectively.

Now we introduce the "trial function" $f\left(\boldsymbol{r}_{1}, \boldsymbol{r}_{2}\right)=\phi\left(\boldsymbol{r}_{1}\right) \psi\left(\boldsymbol{r}_{2}\right)$, where $\psi\left(\boldsymbol{r}_{2}\right)$ is a linear combination of (7) and will be specified below. It is easily seen that

$$
\begin{aligned}
\left(H_{1} f, f\right) & =\left(H_{1} \phi, \phi\right)_{1}(\psi, \psi)_{2}=-(\psi, \psi)_{2}, \\
\left(H_{2} f, f\right) & =(\phi, \phi)_{1}\left(H_{2} \psi, \psi\right)_{2}=\left(H_{2} \psi, \psi\right)_{2}, \\
\left(\boldsymbol{p}_{1} \cdot \boldsymbol{p}_{2} f, f\right) & =\left(\boldsymbol{p}_{1} \phi, \phi\right)_{1} \cdot\left(\boldsymbol{p}_{2} \psi, \psi\right)_{2}=0,
\end{aligned}
$$

and hence that

$$
(W f, f)=\frac{2}{Z} \int\left|\psi\left(\boldsymbol{r}_{2}\right)\right|^{2} d \mathbf{r}_{2} \int\left(\frac{1}{r_{12}}-\frac{1}{r_{2}}\right)\left|\phi\left(\mathbf{r}_{1}\right)\right|^{2} d \mathbf{r}_{1} \leqq 0 .
$$

The last inequality follows from the fact that $\phi\left(\boldsymbol{r}_{1}\right)$ is spherically sym-

(18) See, for example, Kemble, footnote 1, p. 157.

(19) See, for example, Kemble, footnote 1, p. 160. 
metric $\left({ }^{20}\right)$. Thus we obtain by (6)

$$
(\tilde{H} f, f) \leqq\left(H_{2} \psi, \psi\right)_{2}=(\psi, \psi)_{2} .
$$

Let $\mathfrak{M}_{N}^{\prime}$ be the set of all linear combinations of (7) with $n \leqq n_{0}$. The dimension number $N$ of $\mathfrak{M}_{N}^{\prime}$ is

$$
N=\sum_{n=1}^{n_{0}} n^{2}=\frac{1}{6} n_{0}\left(n_{0}+1\right)\left(2 n_{0}+1\right) .
$$

Since $\psi_{n l m}$ are eigenfunctions of $H_{2}$ corresponding to the eigenvalues

$$
-\left(1-Z^{-1}\right)^{2} n^{-2}\left(\leqq-\left(1-Z^{-1}\right)^{2} n_{0}^{-2}\right),
$$

it follows that if $\psi \in \mathfrak{M}_{N}^{\prime}$,

$$
\left(H_{2} \psi, \psi\right)_{2} \leqq-\left(1-Z^{-1}\right)^{2} n_{0}^{-2}(\psi, \psi)_{2} .
$$

Let $\mathfrak{M}_{N}$ be the set of functions of the form

$$
f\left(\boldsymbol{r}_{1}, \boldsymbol{r}_{2}\right)=\phi\left(\boldsymbol{r}_{1}\right) \psi\left(\boldsymbol{r}_{2}\right) \quad\left(\psi \in \mathfrak{M}_{N}^{\prime}\right) .
$$

Then $\mathfrak{M}_{N}$ is also an $N$-dimensional linear manifold, and by (8), (10), and $(f, f)=(\phi, \phi)_{1}(\psi, \psi)_{2}=(\psi, \psi)_{2}$ we have

$$
(\tilde{H} f, f) \leqq-\left\{1+\left(1-Z^{-1}\right)^{2} n_{0}^{-2}\right\}(f, f)
$$

for every $f \in \mathfrak{M}_{N}$. It follows from Lemma 1 that

$$
\operatorname{dim} E(\mu) \geqq N \text { with } \mu=-\left\{1+\left(1-Z^{-1}\right)^{2} n_{0}^{-2}\right\} .
$$

4. Result. By (5) and (11) we have an $N$ and $\mu$ satisfying (1) provided that $n_{0}$ satisfies the inequality

$$
-\left\{1+\left(1-Z^{-1}\right)^{2} n_{0}^{-2}\right\}<-(1-\alpha)^{-1},
$$

and can conclude that $\tilde{H}$ has at least $N$ eigenvalues. Solving (12), we have

$$
n_{0}^{2}<\left(1-Z^{-1}\right)^{2}\left(\alpha^{-1}-1\right) .
$$

In the case of the helium atom, we put $Z=2, \alpha^{-1} \doteqdot 4 \times 1820$ and obtain $n_{0}^{2} \leqq 1820$. Hence we can take $n_{0}=42$ and then $N$ is given by (9) as $N=25585$. Of course there is no particular meaning in these figures, for they are the most conservative estimates. If the nuclear mass is as usual assumed to be infinity, we have $\alpha=0$ and $n_{0}$ and $N$ can be taken arbitrarily large.

$\left.{ }^{(20}\right)$ It is sufficient to show that $\int\left(r_{12}^{-1}-r_{2}^{-1}\right) \mid \phi\left(r_{1}\right){ }^{2} d r_{1} \leqq 0$ and, since $\phi\left(r_{1}\right)$ is spherically symmetric, it is in turn sufficient to show that the surface integral $\int\left(r_{12}^{-1}-r_{2}^{-1}\right) d S_{1}$ taken over the sphere $S_{1}: r_{1}=$ const. is $\leqq 0$. But $\int r_{12}^{-1} d S_{1}$ is equal to the Newtonian potential at the point $r_{2}$ by a uniform mass distribution on $S_{1}$, and it is well known that this is equal to $r_{1}^{-1} \int d S_{1}$ or $r_{2}^{-1} \int d S_{1}$ according as $r_{2} \leqq r_{1}$ or $r_{2}>r_{1}$. Hence we have in any case $\int\left(r_{12}^{-1}-r_{2}^{-1}\right) d S_{1} \leqq 0$. 
These considerations can also be made within each of the subspaces of $\mathfrak{S}$ characterized by definite values of the quantum numbers $l$ and $m$, and lead to the same number $n_{0}=42$. It follows that there are at least $42-l$ eigenvalues for each pair of $l$ and $m$.

Evidently the eigenfunctions we have shown to exist must be either symmetric or antisymmetric with respect to $\boldsymbol{r}_{1}$ and $\boldsymbol{r}_{2}$. If we carry out the above calculation with respect to symmetrized trial functions, we can see that our eigenfunctions are mostly antisymmetric, except that of the ground state which is obviously symmetric. Of course this does not mean that other symmetric eigenfunctions are nonexistent; undoubtedly we have lost them only because our estimation was too rough. It will be possible to recover them if we make more detailed estimation of exchange integrals. But we shall not enter into such details of calculation here.

5. Convergence of the variational method. Since we have shown the existence of eigenfunctions of the Hamiltonian, especially of the ground state solution, the variational method of Hylleraas $\left({ }^{3}\right)$ and others is placed on a firm basis. In fact, it is nothing but an application of our principle (1) which is aimed at getting as small a value for $\mu$ as possible for the fixed value $N=1$ or other small value of $N$, while our aim was to make $N$ as large as possible by a suitable choice of $\mu$.

But it is a different question whether we can expect ultimate convergence of the variational method to the correct eigenvalues and eigenfunctions. This is really a difficult problem, and it seems that a satisfactory answer has not been given. Clearly the result depends essentially on the underlying system of functions.

A sufficient condition for the convergence of the variational method was derived in the previous paper $[\mathrm{I}, \S 10]$ in the case of the general many-particle problem, and the system of Hermite orthogonal functions was shown to satisfy it. But we do not know whether other familiar sets of functions, especially those used by Hylleraas $\left({ }^{3}\right)$, satisfy that condition. Thus we have as yet no decisive proof that his method is convergent to correct results, although it is highly plausible $\left({ }^{21}\right)$.

In conclusion, the writer wishes to thank Professor K. Ochiai for his interest and encouragement. He is indebted also to Professor E. C. Kemble of Harvard University for the publication of this work.

University of ToKyo,

TOKYO, JAPAN.

(21) In the paper of Coolidge and James, footnote 1, the subject is discussed and a proof of the convergence of Hylleraas's method is given, but in the writer's opinion the proof is not complete from the mathematical standpoint, for due attention is not paid to the order of summation in a double series. 